\title{
Association between the overexpression of PBOV1 and the prognosis of patients with hepatocellular carcinoma
}

\author{
CHENGBIAO XUE, ZIBIAO ZHONG, SHAOJUN YE, YANFENG WANG and QIFA YE
}

Institute of Hepatobiliary Diseases of Wuhan University, Transplant Center of Wuhan University, Zhongnan Hospital of Wuhan University, Hubei Key Laboratory of Medical Technology on Transplantation, Wuhan, Hubei 430071, P.R. China

Received January 6, 2017; Accepted May 11, 2018

DOI: $10.3892 / \mathrm{ol} .2018 .9013$

\begin{abstract}
Prostate and breast cancer overexpressed 1 (PBOV1) is a known human protein-coding gene with an uncharacterized function; it has been demonstrated to be overexpressed in a variety of human cancer types. The overexpression of PBOV1 has been indicated as significantly associated with the poor prognosis of these types of cancer. However, the function of PBOV1 in hepatocellular carcinoma (HCC) has not yet been elucidated. The present study was designed to evaluate the expression and prognostic significance of PBOV1 in HCC Reverse transcription-quantitative polymerase chain reaction and western blot analyses were conducted to measure the expression of PBOV1 in HCC cell lines and tissues. The associations between PBOV1 expression and clinicopathological features were statistically analyzed. The association between PBOV1 expression and the prognosis of HCC patients was analyzed by the Kaplan-Meier method. The mRNA and protein expression levels of PBOV1 were significantly increased in the HCC cell lines and HCC tissues (all $\mathrm{P}<0.05$ ) compared with normal cell lines and tissues. In addition, PBOV1 expression was significantly associated with maximal tumor size $(\mathrm{P}=0.032)$, tumor metastasis $(\mathrm{P}=0.035)$ and tumor stage $(\mathrm{P}=0.017)$. The Kaplan-Meier survival curves indicated that overall survival was significantly poorer in patients with HCC with PBOV1 overexpression $(\mathrm{P}<0.05)$ compared with patients with low expression levels. The multivariate analysis indicated that high PBOV1 expression was an independent predictor of poor overall survival. To the best of our knowledge, the data of the present study describes the expression pattern of PBOV1 in
\end{abstract}

Correspondence to: Dr Qifa Ye, Institute of Hepatobiliary Diseases of Wuhan University, Transplant Center of Wuhan University, Zhongnan Hospital of Wuhan University, Hubei Key Laboratory of Medical Technology on Transplantation, 169 Donghu Road, Wuhan, Hubei 430071, P.R. China

E-mail: qifa.ye@outlook.com

Key words: prostate and breast cancer overexpressed 1, hepatocellular carcinoma, prognosis, proliferation
HCC for the first time, and also suggests that PBOV1 may serve as a valuable prognostic biomarker for HCC.

\section{Introduction}

Hepatocellular carcinoma (HCC) is one of the most common malignancies globally. In particular, $\mathrm{HCC}$ is more frequent in developing countries like China compared with in developed countries like the United States of America, as viral hepatitis infections [hepatitis B virus (HBV) and hepatitis $\mathrm{C}$ virus (HCV)] are more common in developing countries (1). Concurrently, the frequency of $\mathrm{HBV}$ is higher compared with HCV infection in China (2). Untreated viral hepatitis infection often leads to chronic liver disease and cirrhosis; in these conditions, almost one-third of patients will ultimately develop HCC over a period of several years (3). In addition, $\mathrm{HCC}$ is most common in men; the primary reason for this may be differences in lifestyle, including alcohol consumption or smoking habits (4). Each year, 500,000 incident cases were diagnosed as HCC globally (4). The treatment options for early-stage HCC include surgical resection and liver transplantation (5). Although early diagnostic markers have been developed in previous decades, the long-term survival of patients with HCC remains poor (6). Therefore, identification of valuable diagnostic markers and novel therapeutic strategies are a major challenge in HCC.

Prostate and breast cancer overexpressed 1 ( $P B O V 1$ ), also termed UROC28 or UC28, is a human protein-coding gene with a 2,501-bp single-exon mRNA and an open reading frame encoding a protein of 135 amino acids (7). The gene was first characterized by An et al (7) in 2000 as being overexpressed at the protein and mRNA levels in prostate, breast and bladder cancer tissues, as well as in the glandular epithelium (7-10). An additional study confirmed that the expression of PBOV1 was increased in prostate cancer compared with adjacent benign epithelium, and PBOV1 overexpression promoted cell proliferation and colony formation ability, and tumorigenic ability in vitro (11). Notably, PBOV1 is poorly conserved in mammalian evolution and is expressed in multiple types of human tumors, but only rarely in normal tissue samples (12). It was also identified that the expression of PBOV1 in prostate cancer cells is upregulated by dihydrotestosterone (12). In addition, PBOV1 transcription 
in breast cancer cells was demonstrated to be downregulated by estradiol in a dose-dependent manner (13), and the mRNA and protein expression of PBOV1 was identified to be upregulated in ovarian cancer cell lines (14). However, there were negative associations between high PBOV1 expression and ascending histological grade and late TNM stage (14). It was also suggested that patients with high PBOV1 expression experience longer overall survival times; and, therefore, it was hypothesized that PBOV1 may serve as a tumor-suppressor gene in ovarian cancer, which is different from previous data regarding prostate, breast and bladder cancer (14). Therefore, additional studies are required to understand the role of PBOV1 in the development of cancer.

Considering the poor prognosis of $\mathrm{HCC}$, and that the role of PBOV1 in cancer remains incompletely characterized, the present study aimed to investigate the expression pattern of PBOV1 in HCC and its association with clinicopathological features for the first time. The effectiveness of PBOV1 as an independent prognostic factor was assessed using multivariate analysis.

\section{Materials and methods}

Patients and frozen tissue samples. A total of 109 patients (54 females and 55 males; age range, $41-75$ years; mean age, 57) with HCC who received treatment between March 2006 and February 2010 at Zhongnan Hospital of Wuhan University were enrolled. Written informed consent was obtained from each patient. A total of 2 patients exhibited IVa stage disease, and the maximum tumor size of these patients was $<13 \mathrm{~cm}$. The tumor stages of all the enrolled patients were classified according to the 7th Tumor-Node-Metastasis (TNM) classification system of the International Union Against Cancer (15). The patients who did not receive percutaneous ablation, radiotherapy or chemoembolization prior to surgery were selected and enrolled. The study was approved and monitored by the Ethics Committee of Zhongnan Hospital of Wuhan University (Hubei, China), and conformed to the guidelines of the Declaration of Helsinki.

Paired tumor and non-tumor liver tissue samples were obtained from all patients immediately following liver resection (16), and snap frozen at $-80^{\circ} \mathrm{C}$ until use. The tissues were collected from the patients during surgery, and the normal tissues were obtained from the patients at an $\sim 5-\mathrm{cm}$ margin from the tumor tissues. The pathological diagnosis was confirmed in all cases by the Department of Pathology, Zhongnan Hospital of Wuhan University. Clinicopathological features of the patients were recorded in the follow-up survey. In addition, the associations between PBOV1 expression and clinicopathological features were analyzed using a $\chi^{2}$ test, and the results are summarized in Table I.

Cell lines and transfection. Two HCC cell lines (HCCLM3 and HEP3B) and one normal liver cell line (L02) were obtained from the American Type Culture Collection (Manassas, VA, USA). These cell lines were cultured in Dulbecco's modified Eagle's medium (Invitrogen; Thermo Fisher Scientific, Inc., Waltham, MA, USA) supplemented with $10 \%$ fetal bovine serum (Invitrogen; Thermo Fisher Scientific, Inc.). All cells were maintained at $37^{\circ} \mathrm{C}$ in a humidified atmosphere containing $5 \% \mathrm{CO}_{2}$ and $95 \%$ air.
Table I. Associations between PBOV1 expression with the clinicopathological features of hepatocellular carcinoma.

\begin{tabular}{|c|c|c|c|c|}
\hline \multirow[b]{2}{*}{ Characteristic } & \multirow[b]{2}{*}{$\mathrm{N}$} & \multicolumn{2}{|c|}{$\begin{array}{c}\text { PBOV1 } \\
\text { expression, } \mathrm{n}\end{array}$} & \multirow[b]{2}{*}{ P-value } \\
\hline & & High & Low & \\
\hline \multicolumn{5}{|l|}{ Sex } \\
\hline Male & 55 & 39 & 16 & NS \\
\hline Female & 54 & 34 & 20 & \\
\hline \multicolumn{5}{|l|}{ Age, years } \\
\hline$\geq 50$ & 62 & 38 & 24 & NS \\
\hline$<50$ & 47 & 35 & 12 & \\
\hline \multicolumn{5}{|c|}{ Maximal tumor size, $\mathrm{cm}$} \\
\hline$\geq 5$ & 67 & 50 & 17 & 0.032 \\
\hline$<5$ & 42 & 23 & 19 & \\
\hline \multicolumn{5}{|c|}{ Serum AFP level, ng/ml } \\
\hline$<400$ & 48 & 29 & 19 & \\
\hline \multicolumn{5}{|l|}{$\mathrm{HBsAg}$} \\
\hline Positive & 50 & 34 & 16 & NS \\
\hline Negative & 59 & 39 & 20 & \\
\hline \multicolumn{5}{|c|}{ Tumor metastasis } \\
\hline No & 58 & 43 & 15 & 0.035 \\
\hline Yes & 51 & 30 & 21 & \\
\hline \multicolumn{5}{|l|}{ TNM stage } \\
\hline I-II & 49 & 27 & 22 & 0.017 \\
\hline III-IV & 60 & 46 & 14 & \\
\hline
\end{tabular}

NS, not significant; PBOV1, prostate and breast cancer overexpressed 1; AFP, $\alpha$-fetoprotein; HBsAg, hepatitis B surface antigen; TNM, Tumor Node Metastasis.

PBOV1 short hairpin RNAs (shRNAs) were designed according to a previous study, and synthesized by Shanghai GeneChem Co., Ltd. (Shanghai, China) (10). The shRNA sequences were as follows: shRNA 1, 5'-CCAGCCAAGTAA CTGAACCAT; and shRNA 2, GCAGACACACTTGACCAT GAA-3'. HCCLM3 and HEP3B cells were transfected with $4 \mu \mathrm{g}$ PBOV1 shRNA using Lipofectamine ${ }^{\circledR} 2000$ (Invitrogen; Thermo Fisher Scientific, Inc.) for $24 \mathrm{~h}$, as described previously (17).

RNA extraction, cDNA synthesis and reverse transcription-quantitative polymerase chain reaction $(R T-q P C R)$. Total RNA was extracted from all cultured cell lines and the 109 tissue samples using TRIzol ${ }^{\circledR}$ reagent (Invitrogen; Thermo Fisher Scientific, Inc.) according to the manufacturer's protocol. Purified RNA was additionally treated with RNase-free DNase I (Sigma-Aldrich; Merck KGaA, Darmstadt, Germany).

The first-strand cDNA was synthesized using a RevertAid First Strand cDNA Synthesis kit (cat. no. K1621; Fermentas; Thermo Fisher Scientific, Inc., Pittsburgh, PA, USA), including oligo(dT) primers. The whole experimental procedure 
A

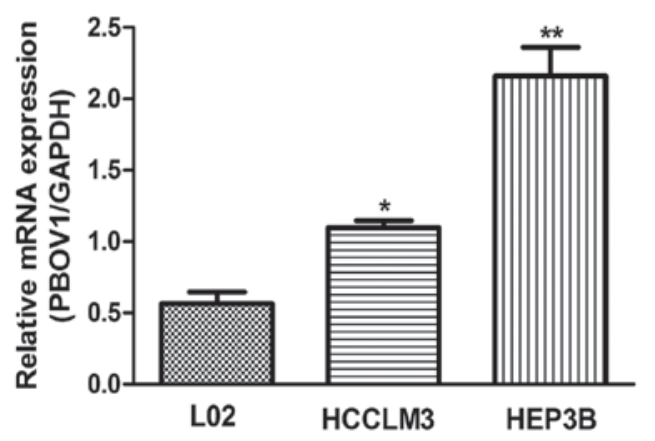

C

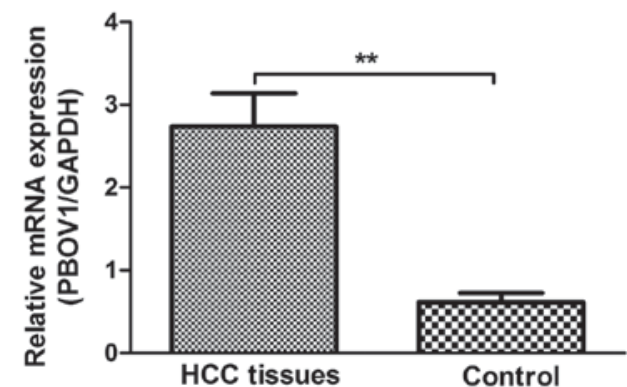

B
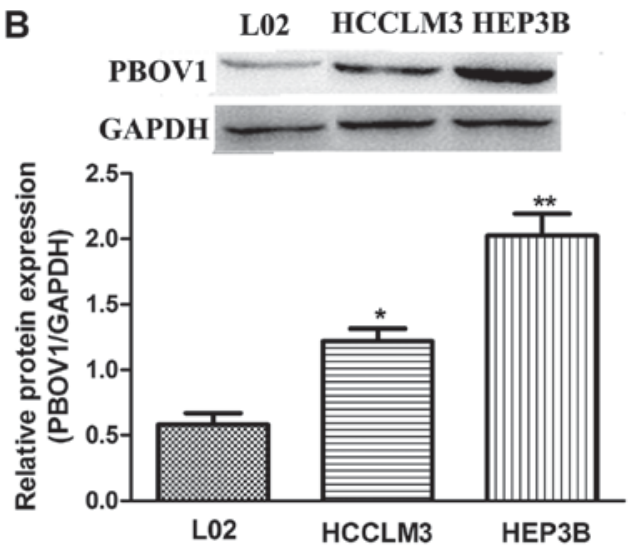

D

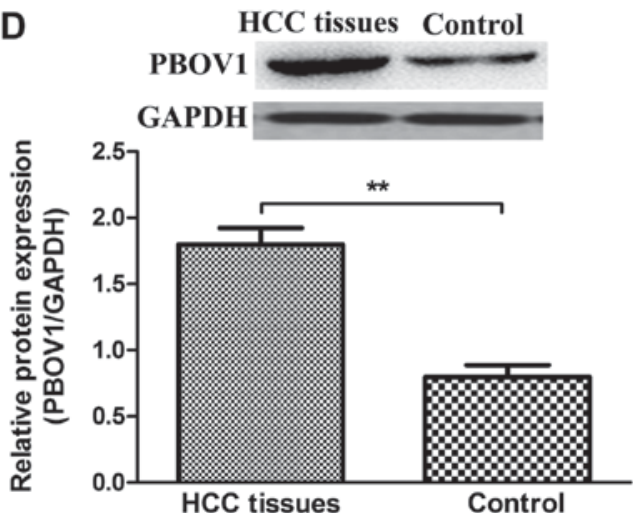

Figure 1. PBOV1 expression in HCC cell lines and tissues. (A) RT-qPCR analysis demonstrated increased PBOV1 mRNA expression levels in HCC cell lines compared with a normal liver cell line (L02). (B) Western blot analysis demonstrated increased PBOV1 protein expression levels in HCC cell lines compared with a normal liver cell line. ${ }^{*} \mathrm{P}<0.05$ and ${ }^{* * *} \mathrm{P}<0.01$ vs. $\mathrm{L} 02$ cells. (C) RT-qPCR analysis demonstrated an increased PBOV1 mRNA expression in HCC tissues compared with normal liver tissues. (D) Western blot analysis demonstrated increased PBOV1 protein expression in HCC tissues compared with normal liver tissues. ${ }^{* *} \mathrm{P}<0.01$. PBOV1, prostate and breast cancer overexpressed 1; HCC, hepatocellular carcinoma; RT-qPCR, reverse transcription-quantitative polymerase chain reaction.

was performed following the manufacturer's protocol. The resulting cDNA was stored at $-20^{\circ} \mathrm{C}$ until use.

RT-qPCR was performed using an ABI Prism 7500 Sequence Detection system (Applied Biosystems; Thermo Fisher Scientific, Inc.) and SYBR-Green I (Invitrogen; Thermo Fisher Scientific, Inc.). PCR primers targeting the PBOV1 coding sequence were designed based on GenBank cDNA AF189270 (https://www.ncbi.nlm.nih. gov/gene/?term=AF189270). PBOV1-specific primers used were as follows: Forward, 5'-AAGGAACCAGAAATA TGAGG-3' and reverse, 5'-TTTGGATAAGTAGAGAAG AC-3'. The amplification was performed in the following conditions: $1 \mathrm{~min}$ at $95^{\circ} \mathrm{C}$, then 35 cycles of $30 \mathrm{sec}$ at $95^{\circ} \mathrm{C}$, $30 \mathrm{sec}$ at $58^{\circ} \mathrm{C}$ and $40 \mathrm{sec}$ at $72^{\circ} \mathrm{C}$, and a final elongation step at $72^{\circ} \mathrm{C}$ for $5 \mathrm{~min}$. The housekeeping gene GAPDH was used as an internal control to normalize the gene expression data. The GAPDH-specific primers used were as follows: Forward, 5'-TGA AGGTCGGAGTCAACGGATTTGGT-3' and reverse, 5'-CATGTGGGCCATGAGGTCCACCAC-3'. The same PCR conditions were used. The expression level of PBOV1 was calculated as $2^{-[(\mathrm{Cq} \text { of PBOV1)-(Cq of GAPDH)] }}$, where $\mathrm{Cq}$ represents the quantification cycle value for each transcript (18). Experiments were repeated in triplicate to ensure accuracy. The levels of PBOV1 were used to classify these patients into high or low PBOV1 expression group (threshold value, 1.05).
Western blot assay. Total proteins were extracted from 109 fresh tissue pairs and the cultured cells using a Total Protein Extraction kit (cat. no. KGP2100) with protease inhibitor, phosphatase inhibitor, and PMSF (Nanjing Keygen Biotech, Co., Ltd., Nanjing, China), according to the manufacturer's protocol. The protein concentration was measured using a Pierce BCA Protein Assay kit (Thermo Fisher Scientific, Inc.). Equal amounts $(50 \mu \mathrm{g})$ of each protein sample were loaded and separated via $10 \%$ SDS-PAGE. Then, the samples were transferred onto a PVDF membrane, and the membrane was blocked with $5 \%$ fat-free milk at room temperature for $2 \mathrm{~h}$. The membrane was probed with an anti-PBOV1 antibody (1:1,000 dilution; cat. no. ab70018) and an anti-GAPDH antibody (1:1,000 dilution; cat. no. ab181602; both Abcam, Cambridge, MA, USA) at $4^{\circ} \mathrm{C}$ overnight. Expression of PBOV1 was determined with a horseradish peroxidase-conjugated anti-rabbit $\operatorname{IgG}(1: 3,000$ dilution; cat. no. ab6721; Abcam) at room temperature for $2 \mathrm{~h}$. The signals were developed using SuperSignal West Pico PLUS (Pierce; Thermo Fisher Scientific, Inc.) and quantified using Bio-Rad Gel Doc EZ system with the Image Lab 3.0 software (Bio-Rad Laboratories, Inc., Hercules, CA, USA), according to the manufacturer's protocol (19). Experiments were repeated in triplicate to ensure accuracy.

MTT assay. The effect of PBOV1 on cell proliferation was assessed with an MTT assay (Sigma-Aldrich; Merck KGaA), 
A

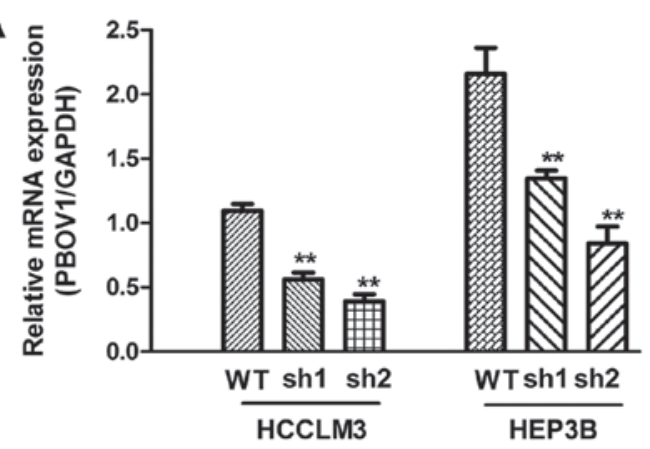

C

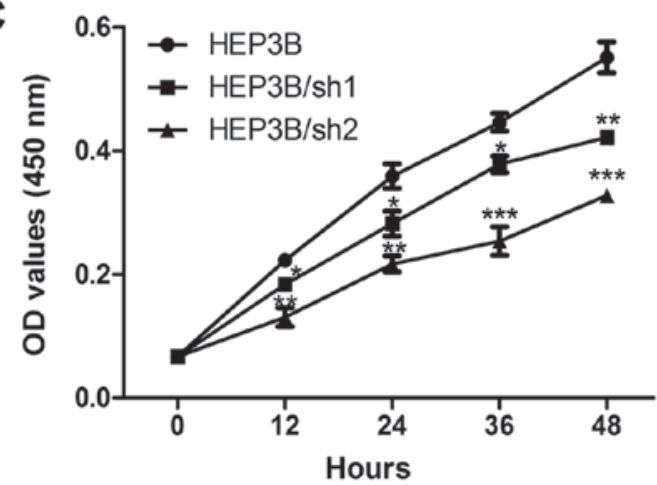

B
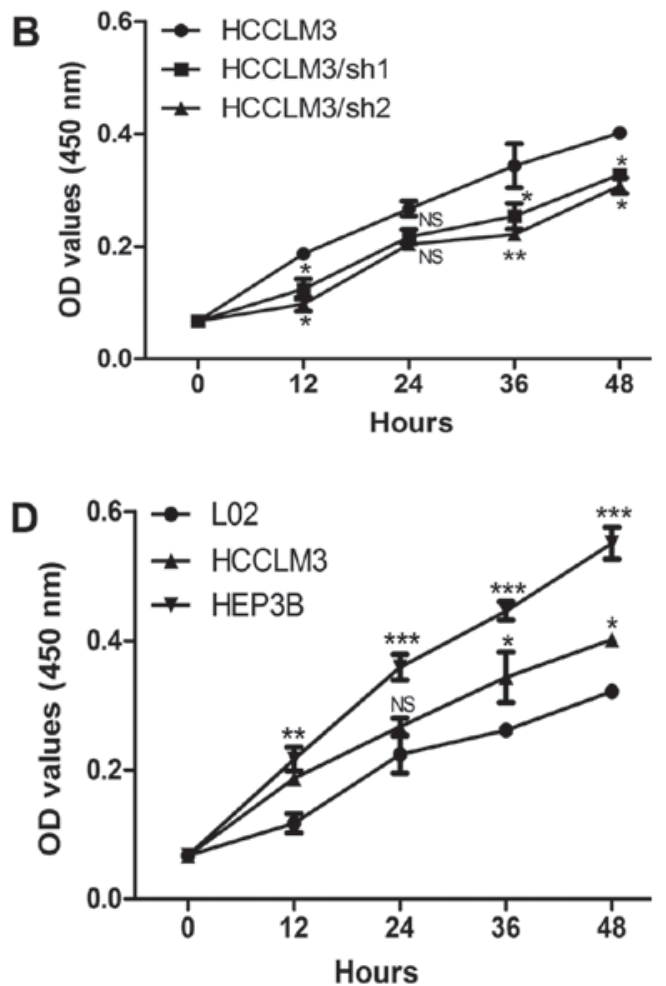

Figure 2. Effect of PBOV1 on the cell proliferation of HCC cell lines. (A) shRNA transfection to downregulate the expression of PBOV1 on HCC cell lines. (B) MTT assay demonstrated that the cell proliferation of HCCLM3 cell lines with PBOV1-specific shRNA transfection was significantly decreased compared with the WT HCCLM3 cell line. (C) MTT assay demonstrated that the cell proliferation of HEP3B cell lines with PBOV1-specific shRNA transfection was significantly decreased compared with the WT HEP3B cell line. (D) MTT assay demonstrated that the cell proliferation of HCC cell lines was significantly decreased compared with the normal liver cell line. ${ }^{*} \mathrm{P}<0.05,{ }^{* *} \mathrm{P}<0.01$ and ${ }^{* * * *} \mathrm{P}<0.001$. PBOV1, prostate and breast cancer overexpressed $1 ;$ HCC, hepatocellular carcinoma; sh, short hairpin; WT, wild-type; NS, not significant; OD, optical density.

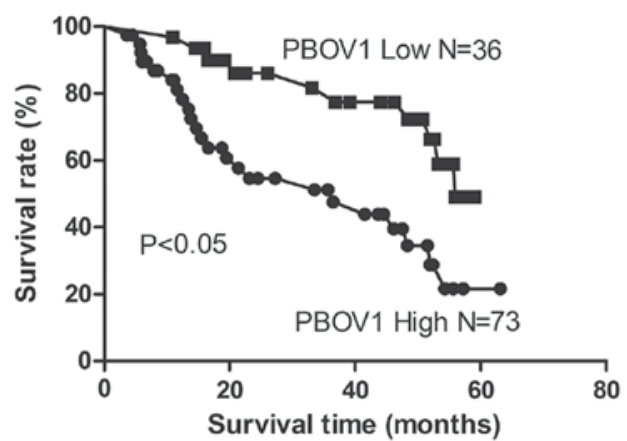

Figure 3. Survival rates of patients with high and low PBOV1 expression levels. Kaplan-Meier analysis revealed a significantly poorer overall survival rate in patients with high PBOV1 expression compared with those with low expression. PBOV1, prostate and breast cancer overexpressed 1.

as described previously (20). Cells were seeded into 96-well culture plates at a density of $1 \times 10^{4} /$ well. At various time points $(12,24,36$ and $48 \mathrm{~h}), 10 \mu \mathrm{l}(50 \mu \mathrm{g})$ MTT was added to each well and incubated for $4 \mathrm{~h}$ at $37^{\circ} \mathrm{C}$. Next, the medium was removed, and $150 \mu \mathrm{l}$ dimethyl sulfoxide (Sigma-Aldrich; Merck KGaA) was added to dissolve the resultant formazan crystals. Absorbance was measured using a microplate reader at $450 \mathrm{~nm}$. All experiments were performed in triplicate.

Statistical analysis. All statistical analyses were performed using the SPSS 10.0 statistical software package (SPSS, Inc., Chicago, IL, USA). The data was presented as mean \pm standard deviation. The associations between PBOV1 expression and clinicopathological characteristics were analyzed using a $\chi^{2}$ test. The survival probability was calculated using the Kaplan-Meier method, and the statistical differences were examined using a log-rank test. Multivariate analysis was performed to assess the variables deemed significant in univariate analyses, each performed using a Cox proportional hazards regression model. Differences between two groups were calculated using a Student's t-test. One-way analysis of variance and Tukey's post-hoc test were performed to analyze the statistical differences between $\geq 3$ groups. $\mathrm{P}<0.05$ was considered to indicate a statistically significant difference.

\section{Results}

PBOVI is upregulated in HCC cell lines and tissues. The mRNA and protein levels of PBOV1 were examined in L02, HCCLM3 and HEP3B cell lines using RT-qPCR and western blot analysis, respectively. The data indicated that the PBOV1 expression levels (mRNA and protein) in the HCC cell lines were significantly increased compared with in the normal liver L02 cell line ( $\mathrm{P}<0.05$; Fig. 1A and B). Of the HCC cell lines investigated the HEP3B cell line exhibited the highest mRNA and protein PBOV1 expression levels. Collectively, these results suggest that PBOV1 is upregulated in HCC cell lines.

To investigate whether PBOV1 may be involved in the progression of $\mathrm{HCC}$, the mRNA and protein expression levels of PBOV1 in all the collected HCC tissues and their 
Table II. Univariate and multivariate analyses of overall survival.

\begin{tabular}{|c|c|c|c|c|c|c|}
\hline \multirow[b]{2}{*}{ Variables } & \multicolumn{3}{|c|}{ Univariate analysis } & \multicolumn{3}{|c|}{ Multivariate analysis } \\
\hline & HR & $95 \% \mathrm{CI}$ & P-value & HR & $95 \% \mathrm{CI}$ & P-value \\
\hline PBOV1 & 2.416 & $1.176-4.962$ & 0.016 & 2.142 & $1.146-4.004$ & $0.010^{\mathrm{a}}$ \\
\hline Age & 1.604 & $0.869-2.960$ & 0.131 & 1.714 & $0.888-3.310$ & 0.108 \\
\hline Sex & 1.666 & $0.897-3.095$ & 0.106 & 1.613 & $0.828-3.140$ & 0.160 \\
\hline Maximal tumor size & 2.262 & $1.112-4.599$ & 0.024 & 2.253 & $1.165-4.358$ & $0.015^{\mathrm{a}}$ \\
\hline Serum AFP level & 1.950 & $0.960-3.961$ & 0.065 & 1.824 & $0.952-3.494$ & 0.070 \\
\hline HBsAg & 1.838 & $0.975-3.462$ & 0.059 & 1.746 & $0.918-3.320$ & 0.089 \\
\hline Tumor metastasis & 2.083 & $1.036-4.188$ & 0.039 & 2.140 & $1.097-4.176$ & $0.026^{\mathrm{a}}$ \\
\hline TNM stage & 2.029 & $1.030-3.996$ & 0.040 & 1.912 & $1.012-3.612$ & $0.046^{\mathrm{a}}$ \\
\hline
\end{tabular}

${ }^{a} \mathrm{P}<0.05$. HR, hazard ratio; CI, confidence interval; PBOV1, prostate and breast cancer overexpressed 1; AFP, $\alpha$-fetoprotein; HBsAg, hepatitis B surface antigen; TNM, Tumor Node Metastasis.

paired adjacent noncancerous tissues were determined. It was observed that the expression of PBOV1 (mRNA and protein) was significantly upregulated in the HCC tissues when compared with in the matched noncancerous tissues $(\mathrm{P}<0.01$; Fig. 1C and D). Among the 109 patients, 73 patients (66.97\%) exhibited higher PBOV1 expression levels in tumor tissues compared with in the matched noncancerous tissues, and, thus, these 73 patients were assigned to the high PBOV1 expression group. The remaining 36 patients who exhibited lower PBOV1 expression levels were assigned to the low PBOV1 expression group. These data demonstrate the upregulated status of PBOV1 in HCC; and it was hypothesized that PBOV1 may serve an important role in the progression of HCC.

PBOV1 promotes proliferation of $H C C$ cell lines. In order to assess the oncogenic activity of PBOV1 in HCC, a stable PBOV1-knockdown HCC cell line was established using specific shRNA. The PBOV1 mRNA expression level of these transfected cell lines was assessed by RT-qPCR. As expected, the transfection of the PBOV1-shRNA into the HCC cell lines resulted in significantly decreased PBOV1 mRNA expression levels when compared with the cell lines without shRNA transfection (all $\mathrm{P}<0.01$; Fig. 2A). Furthermore, the proliferation rates of these cell lines were measured using an MTT assay. The suppression of PBOV1 expression significantly inhibited the proliferation of the HCCLM3 cell line in all the time points selected except in $24 \mathrm{~h}(\mathrm{P}<0.05$; Fig. 2B). However, the cell proliferation rate of HEP3B was inhibited by shRNAs in all the time points selected (all $\mathrm{P}<0.05$; Fig. 2C) The proliferation rates of $\mathrm{HCC}$ and normal liver cell lines were also compared. The results in Fig. 2D indicated that the cell proliferation rates of the HEP3B cell line were significant increased compared with the normal liver cell line in all the time points selected ( $\mathrm{P}<0.05)$. Additionally, the cell proliferation rates of HCCLM3 cell line was higher than normal liver cell line in all the time points selected except in $24 \mathrm{~h}(\mathrm{P}<0.05$; Fig. 2D). Taken together, these results suggest the PBOV1 expression is an important regulator of HCC cell proliferation.
Association between PBOVI expression and clinicopathological features. In order to analyze the associations between PBOV1 expression and the clinicopathological characteristics of patients with HCC, the 109 patients were divided into two groups according to the PBOV1 expression level. The clinicopathological features were collected from all 109 patients and summarized in Table I. A $\chi^{2}$ test was conducted to evaluate the association between PBOV1 expression and all the clinicopathological features. As indicated in Table I, PBOV1 expression was significantly associated with maximal tumor size $(\mathrm{P}=0.032)$, tumor metastasis $(\mathrm{P}=0.035)$, and tumor stage $(\mathrm{P}=0.017)$, while no significant associations between PBOV1 expression and other variables, such as age, sex, hepatitis B surface antigen (HBsAg) and serum AFP, were observed (all $\mathrm{P}>0.05$ ).

Prognostic value of high PBOVI expression in HCC. The association between PBOV1 expression and the survival of patients with HCC following surgery was investigated using the Kaplan-Meier analysis and log-rank test. As demonstrated in Fig. 3, patients with high PBOV1 expression levels exhibited a significantly shorter survival time following surgery compared with patients with low PBOV1 expression levels $(\mathrm{P}<0.05)$. Univariate analysis revealed that among the collected clinicopathological features, maximal tumor size, tumor metastasis, tumor stage and PBOV1 expression were significantly associated with the overall survival of HCC patients (all $\mathrm{P}<0.05$; Table II), while the other features were not statistically significant prognosticators (all P>0.05; Table II). Multivariate analysis using the Cox proportional hazards model for all variables identified that PBOV1 expression was a significant independent predictor of poor prognosis in patients with $\mathrm{HCC}$ in addition to maximal tumor size, tumor metastasis and tumor stage (all $\mathrm{P}<0.05$; Table II).

\section{Discussion}

Previous data have demonstrated that one prominent feature of various tumor types is the abundant upregulation of various transcripts, a number of which have an uncharacterized function (21-23). The PBOVI is gene encoding a protein of 
135 amino acids in length, located at chromosome 6q23-24 region (7). $P B O V 1$ has been identified to be overexpressed in a number of types of human cancer, including ovarian (14), breast (13), endometrial and prostate cancer (11). Previous studies have demonstrated that overexpression of PBOV1 promoted cell proliferation, cell cycle progression and tumorigenicity in vitro, whereas the knockdown of PBOV1 reduced these effects $(11,14)$. However, whether or not PBOV1 has similar expression pattern in $\mathrm{HCC}$ as these aforementioned cancer types remains unknown.

Therefore, it is required to examine the expression level of PBOV1 in HCC, in order to additionally investigate whether or not PBOV1 participates in the progression of HCC. The expression of PBOV1 in one normal liver cell line and two HCC cell lines were examined, and the results demonstrated that PBOV1 was significantly upregulated in the HCC cell lines, compared with in the normal cell line. Considering this, a total of 109 paired HCC tumor tissues and adjacent noncancerous tissues were collected. It was identified that, in comparison with the adjacent noncancerous in tissues, PBOV1 mRNA and protein levels were upregulated in HCC tumor tissues. These data in HCC were the same as the results from the analyses in ovarian, breast, endometrial and prostate cancer, and suggest that PBOV1 may serve a role in the tumorigenesis of HCC. However, how PBOV1 regulates HCC progression requires additional exploration.

Using PBOV1-specific shRNA, a stable PBOV1-knockdown HCC cell line was created. The PBOV1 mRNA expression data revealed the successful PBOV1 knockdown in the selected $\mathrm{HCC}$ cell lines. Then, the cell proliferation rate analysis results indicated that the introduction of PBOV1 shRNA significantly downregulated the cell proliferation rates. Concurrently, the normal liver cell line, with the lowest PBOV1 expression among all the cell lines used, also exhibited the lowest cell proliferation rate. Therefore, the results of the present study suggested that PBOV1 overexpression may promote HCC cancer cell proliferation and described a potential mechanism of how PBOV1 promotes tumorigenesis in HCC; however, the detailed mechanism requires additional study. A previous study investigating the effect of PBOV1 on prostate cells revealed that the overexpression of PBOV1 suppressed cell cycle inhibitors cyclin-dependent kinase inhibitor 1 and cyclin-dependent kinase inhibitor 1B, increased retinoblastoma protein phosphorylation levels and cyclin D1 expression and suggested that PBOV1 contributed to $\mathrm{G}_{1} / \mathrm{S}$ transition (11), which may also be a mechanism for the proliferation-stimulatory effect of PBOV1.

The 109 archived paraffin-embedded HCC tumor tissues were additionally divided into two groups according to the PBOV1 expression level, and the association of PBOV1 expression with the clinicopathological features was analyzed. Notably, it was identified that the expression of PBOV1 in HCC was closely associated with well-known tumor malignancy indicators, including tumor size, tumor metastasis and tumor stage. Concurrently, whether or not the HBV infection was associated with PBOV1 expression, as HBV infection is a major cause of HCC, was analyzed. However, it was demonstrated that the PBOV1 expression was not associated with $\mathrm{HBsAg}$, and therefore it was hypothesized that there was no association between HBV infection and PBOV1 expression level. Then, the association between PBOV1 expression and survival duration was assessed using a Kaplan-Meier analysis. The log-rank test indicated that PBOV1 expression level was inversely associated with overall survival of the patients with HCC. Next, the multivariate Cox proportional hazards model suggested that PBOV1 expression was an independent predictor for the overall survival duration of patients with HCC.

In conclusion, PBOV1 overexpression is a common feature in patients with HCC. Additionally, the present study provides the clinical evidence that PBOV1 is an independent prognostic factor for the outcome of patients with HCC. However, the present study had certain limitations, including the fact that the associations between HCV infection, degree of hepatitis, liver cirrhosis and the expression of PBOV1 were not analyzed. Independent validation of these clinical data and additional investigation of the detailed effects of PBOV1 on cell behaviors are required. Nevertheless, the present study provides a basis for the development of a novel diagnostic and prognostic biomarker for HCC.

\section{Acknowledgements}

Not applicable.

\section{Funding}

The present study was supported by National Natural Science Foundation of China-Xinjiang Joint Fund (grant no. U1403222).

\section{Availability of data and materials}

The datasets used and/or analyzed during the current study are available from the corresponding author on reasonable request.

\section{Authors' contributions}

CBX, ZBZ, SJY, YFW and QFY conceived and designed the experiments, performed the experiments, and analyzed the data. CBX and QFY wrote the paper.

\section{Ethics approval and consent to participate}

The study was approved and monitored by the Ethics Committee of Zhongnan Hospital of Wuhan University, and conformed to the ethical guidelines of the Helsinki Declaration. Written informed consent was obtained from all the enrolled patients.

\section{Patient consent for publication}

Not applicable.

\section{Competing interests}

The authors declare that they have no competing interests.

\section{References}

1. Chen W, Zheng R, Baade PD, Zhang S, Zeng H, Bray F, Jemal A, Yu XQ and He J: Cancer statistics in China, 2015. CA Cancer J Clin 66: 115-132, 2016.

2. Cui Y and Jia J: Update on epidemiology of hepatitis B and C in China. J Gastroenterol Hepatol 28 (Suppl 1): S7-S10, 2013. 
3. Fattovich G, Stroffolini T, Zagni I and Donato F: Hepatocellular carcinoma in cirrhosis: Incidence and risk factors. Gastroenterology 127 (5 Suppl 1): S35-S50, 2004.

4. Forner A, Llovet JM and Bruix J: Hepatocellular carcinoma. Lancet 379: 1245-1255, 2012

5. Block TM, Mehta AS, Fimmel CJ and Jordan R: Molecular viral oncology of hepatocellular carcinoma. Oncogene 22: 5093-5107, 2003.

6. Maluccio M and Covey A: Recent progress in understanding, diagnosing, and treating hepatocellular carcinoma. CA Cancer J Clin 62: 394-399, 2012.

7. An G, Ng AY, Meka CS, Luo G, Bright SP, Cazares L, Wright GL Jr and Veltri RW: Cloning and characterization of UROC28, a novel gene overexpressed in prostate, breast, and bladder cancers Cancer Res 60: 7014-7020, 2000.

8. Loizidou MA, Cariolou MA, Neuhausen SL, Newbold RF, Bashiardes E, Marcou Y, Michael T, Daniel M, Kakouri E, Papadopoulos $\mathrm{P}$, et al: Genetic variation in genes interacting with BRCA $1 / 2$ and risk of breast cancer in the Cypriot population. Breast Cancer Res Treat 121: 147-156, 2010.

9. Samusik N, Krukovskaya L, Meln I, Shilov E and Kozlov AP: PBOV1 is a human de novo gene with tumor-specific expression that is associated with a positive clinical outcome of cancer. PLoS One 8: e56162, 2013.

10. Doak SH, Jenkins SA, Hurle RA, Varma M, Hawizy A, Kynaston HG and Parry JM: Bone morphogenic factor gene dosage abnormalities in prostatic intraepithelial neoplasia and prostate cancer. Cancer Genet Cytogenet 176: 161-165, 2007.

11. Pan T, Wu R, Liu B, Wen H, Tu Z, Guo J, Yang J and Shen G PBOV1 promotes prostate cancer proliferation by promoting G1/S transition. Onco Targets Ther 9: 787-795, 2016.

12. Krukovskaia LL, Samusik ND, Shilov ES, Polev DE and Kozlov AP: Tumor-specific expression of PBOV1, a new gene in evolution. Vopr Onkol 56: 327-332, 2010 (In Russian).

13. Kamagata C, Tsuji N, Kondoh K, Sasaki M, Kobayashi D, Yagihashi A and Watanabe N: Enhanced expression of the UROC28 gene in human breast cancer: Relationship to ERBB2 gene expression. Anticancer Res 22: 4087-4091, 2002.
14. Wang L, Niu CH, Wu S, Wu HM, Ouyang F, He M and He SY: PBOV1 correlates with progression of ovarian cancer and inhibits proliferation of ovarian cancer cells. Oncol Rep 35: 488-496, 2016

15. Edge SB, Byrd DR, Compton CC, Fritz AG, Greene FL and Trotti A: AJCC cancer staging manual. 7th ed. Springer: New York: pp191-200, 2010.

16. Yamashita Y, Taketomi A, Shirabe K, Aishima S, Tsuijita E, Morita K, Kayashima H and Maehara Y: Outcomes of hepatic resection for huge hepatocellular carcinoma ( $\geq 10 \mathrm{~cm}$ in diameter). J Surg Oncol 104: 292-298, 2011.

17. Hahn WC, Dessain SK, Brooks MW, King JE, Elenbaas B Sabatini DM, DeCaprio JA and Weinberg RA: Enumeration of the simian virus 40 early region elements necessary for human cell transformation. Mol Cell Biol 22: 2111-2123, 2002.

18. Livak KJ and Schmittgen TD: Analysis of relative gene expression data using real-time quantitative PCR and the 2(-Delta Delta C(T)) method. Methods 25: 402-408, 2001.

19. Li J, Zhang N, Song LB, Liao WT, Jiang LL, Gong LY, Wu J, Yuan J, Zhang HZ, Zeng MS and Li M: Astrocyte elevated gene-1 is a novel prognostic marker for breast cancer progression and overall patient survival. Clin Cancer Res 14: 3319-3326, 2008.

20. Song L, Wang L, Li Y, Xiong H, Wu J, Li J and Li M: Sam68 up-regulation correlates with, and its down-regulation inhibits, proliferation and tumourigenicity of breast cancer cells. J Pathol 222: 227-237, 2010

21. Ma W, Yu Q, Jiang J, Du X, Huang L, Zhao L and Zhou QI: miR-517a is an independent prognostic marker and contributes to cell migration and invasion in human colorectal cancer. Oncol Lett 11: 2583-2589, 2016.

22. Wang G, Huang J, Zhu H, Ju S, Wang H and Wang X: Overexpression of GRO- $\beta$ is associated with an unfavorable outcome in colorectal cancer. Oncol Lett 11: 2391-2397, 2016.

23. Tang B, Tang F, Wang Z, Qi G, Liang X, Li B, Yuan S, Liu J, $\mathrm{Yu} \mathrm{S}$ and He S: Overexpression of CTNND1 in hepatocellular carcinoma promotes carcinous characters through activation of Wnt/ß-catenin signaling. J Exp Clin Cancer Res 35: 82, 2016. 